\title{
Path Planning Algorithm with Thinning Preprocess
}

\author{
Wang Yang, He-Gen Xu \\ College of Electronics and Information Engineering, \\ Tongji University, \\ Shanghai, China \\ E-mail:1433346@tongji.edu.cn,xuhegen@tongji.edu.cn
}

\begin{abstract}
This paper proposes path planning algorithm with thinning preprocess to increase the efficiency of path planning as well as reserving the key points (the start point and the target point). A modified Zhang's fast parallel thinning algorithm is proposed with the key points (start point, the target point) reserved, and the $A^{*}$ algorithm is taken as an example for path planning on the grid map. Simulation results show that the algorithm in this paper greatly decreases the search time of $A^{*}$ algorithm while ensuring a reasonable path for mobile robots.
\end{abstract}

Keywords-path planning; $A^{*}$; Zhang's fast parallel thinning algorithm

\section{INTRODUCTION}

Path planning of mobile robot is mainly to solve the problem: finding an optimal path (such as using shorter time or shorter distance) for the mobile robot [1] to traverse between start and target positions in the environment with obstacles. Grid method is one of the main methods of environmental modeling for path planning.

There are many kinds of algorithms to be used on grid map for path planning, such as genetic algorithm, Dijkstra algorithm, A* algorithm, ant colony algorithm, etc. A* algorithm [2] is a kind of optimal priority heuristic search method and there is always a good result in its application. But generally the search time is long and sometimes it maybe even need several minutes, which seriously limits its application in time-critical scenarios. Genetic algorithm [3] has the advantages of adaptive global optimization and probability search, and the disadvantages of poor local search ability and easy to be premature. So it can't guarantee the reliability and efficiency of the robot path planning. Ant colony algorithm has the advantage of strong robustness [4], but it has a slow convergence rate, and is easy to fall into the local optimal solution of the search. Therefore, it is unable to find the global optimal path.

The searching efficiency of the preceding algorithms severely depends on the size of the grid map. Some improved methods have been presented to speed up the time of path planning to some extent, but the efficiency still goes worse with the map size. In this paper, a path planning algorithm with thinning processing is proposed to reduce the number of searching points while maintain the skeleton of the path. The first step is to thin the grid map [5] [6] [7] while reserving the key points (such as start point, target point) which is necessary for path planning, and then followed by a path planning algorithm.
This paper is divided into three parts: in Section 2, thinning process and modification of thinning algorithm is introduced; in Section 3, A* algorithm is introduced; Results and analysis of the simulation output are presented in Section 4.The conclusions drawn from this work are finally presented in Section 5.

\section{THINNING PROCESS}

There are many methods of thinning process, such as Hilditch thinning algorithm [8], OPTA thinning algorithm [9], Zhang's fast parallel thinning algorithm [10] and so on. The modified Zhang's fast parallel thinning algorithm is applied in this paper due to the advantages of fast speed and keeping the connectivity of the curve while reserving some key points necessary for path planning.

\section{A. Zhang's Fast Parallel Thinning Algorithm}

In this algorithm, target points are marked as 1 and background points as 0 . Take a grid of $3 * 3$ as an example, $\mathrm{P}_{1}$ as the center, $\mathrm{P}_{2}$ to $\mathrm{P}_{9}$ as the 8 neighbors of $\mathrm{P}_{1}$, which are shown in Figure1.

The algorithm is mainly divided into 3 steps:

1) At first, mark the points that satisfy these conditions.
a) $2<=\mathrm{N}\left(\mathrm{P}_{1}\right)<=6$;
b) $\mathrm{S}\left(\mathrm{P}_{1}\right)=1$;
c) $\mathrm{P}_{2} \times \mathrm{P}_{4} \times \mathrm{P}_{6}=0$;
d) $\mathrm{P}_{4} \times \mathrm{P}_{6} \times \mathrm{P}_{8}=0$;

Where, $N\left(P_{1}\right)$ is the number of non-zero neighbors for $P_{1}, S\left(P_{1}\right)$ is the number of $0 \rightarrow 1$ pattern in the sequence $P_{2}, P_{3}, P_{4}, P_{5}, P_{6}, P_{7}, P_{8}, P_{9}$.

\begin{tabular}{|c|c|c|}
\hline $\mathrm{P}_{9}$ & $\mathrm{P}_{2}$ & $\mathrm{P}_{3}$ \\
\hline $\mathrm{P}_{8}$ & $\mathrm{P}_{1}$ & $\mathrm{P}_{4}$ \\
\hline $\mathrm{P}_{7}$ & $\mathrm{P}_{6}$ & $\mathrm{P}_{5}$ \\
\hline
\end{tabular}

Figure 1. 8 Neighborhood definition

2) With the above steps: just replace the last two conditions with $\mathrm{P}_{2} \times \mathrm{P}_{4} \times \mathrm{P}_{8}=0$ and $\mathrm{P}_{2} \times \mathrm{P}_{6} \times \mathrm{P}_{8}=0$.

3) The above two steps constitute an iteration, until there is no point to meet the condition of marking in step 1.: At that time, the area composed of the remaining points is the skeleton after this thinning algorithm. 


\section{B. Modified Algorithm}

Zhang's fast parallel thinning algorithm shows a good result for map thinning process, but it is possible that some of the valid points such as the start point and the target point may be also thinned out if these points are non- skeleton points of the path. This will result in a failure of path planning. Thus another condition shall be added to the iteration process of Zhang's fast parallel thinning algorithm to reserve the key point and keep the connectivity of these key points. Figure 2 and Figure 3 show the results of the improved algorithm: Figure 2(a) is the original image and the points with red points are that need to be kept. Figure 2 (b) is the image after thinning process. Figure 2 shows that the modified algorithm is able to reserve the specified points correctly, and ensure the connectivity of the specified points. But some burr defects may appear on the thinned skeleton with the increasing width of the path as shown in Figure 3.

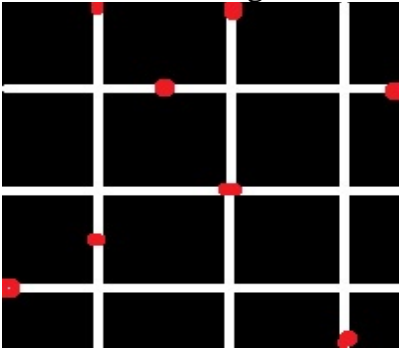

Figure 2(a).

Figure 2(b).

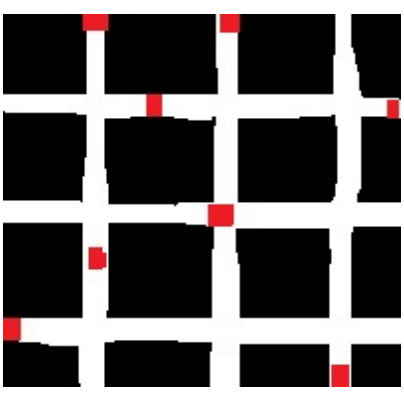

Figure 3(a)

Figure 3(b)

\section{INTRODUCTION OF A*}

A* algorithm is based on the Dijkstra algorithm and BFS algorithm. The heuristic function is used in $A^{*}$ algorithm which defined as:

$$
f(n)=g(n)+h(n)
$$

Where:

- $\mathrm{f}(\mathrm{n})$ is the cost from the initial point to the target point through point $n$.

- $\quad g(n)$ represents the actual cost from the start point to the specified point $n$.

- $\quad h(n)$ represents the estimated cost from the specified point $n$ to the target point.
The value of $h(n)$ is the critical factor for the algorithm. Set $d(n)$ with the actual distance between the specified point $\mathrm{n}$ and the target point. And the conclusions are shown as below:

- if $h(n)<d(n)$, it will search more points and the search time will be longer. But we can get the optimal solution.

- if $h(n)=d(n)$, this is the ideal situation. Expected distance is exactly the same as the actual distance. At this time, the search efficiency is high enough, and the shortest path will be found.

- if $h(n)>d(n)$, the number of points to be searched becomes less and search time is reduced, but it can't guarantee that we can get the optimal solution.

\section{VERIFICATION}

Two groups of simulation are applied to verify the performance of the proposed path planning algorithm. Group A proves the validity of the algorithm in different sizes of grid map. Group B proves the validity of the algorithm in the different complexity of the grid map.

In order to show the reliability of the simulation results, the start points and target points in the two simulation groups are in the similar position of the corresponding map. The size of the simulation pictures are not the same so that the coordinate of the start point and the target point will be different.

\section{A. Simulation -1: The Different Sizes of Grid Map}

As shown in Figure 4, the white points indicate that the points are free and the black points indicate that the points are block. The grid maps are saved as pictures with the size of $200 * 200,400 * 400,800 * 800$.

Thinned grid map which is marked in red, added to the original map is shown in Figure 4.

An example of planning result in original map and thinned map are shown in Figure 5 and Figure 6, respectively. The start points are in red.

This proves the feasibility of the path planning algorithm. In this section, this paper explains the advantage of the path planning algorithm with thinning preprocess, in the aspect of search time on different sizes of grid map. Comparison of the planned path before (in white) and after (in red) thinning process is shown in the Figure 7.

As shown in Table I , it contrasts the path planning time and point on the path with the algorithm before and after thinning.

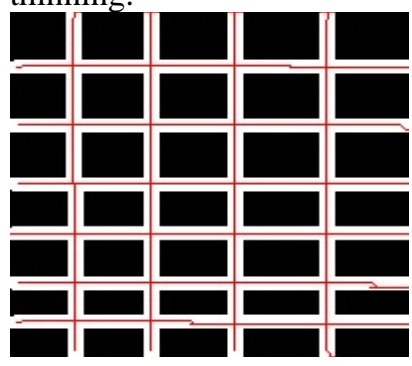

Figure 4. Original image

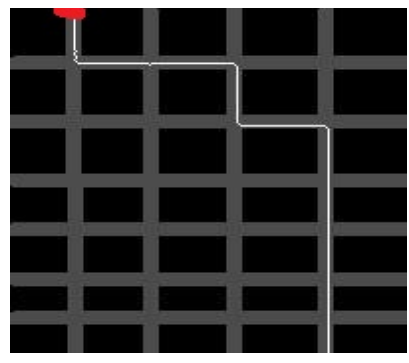

Figure 5. Path in the original image 
TABLE I. PATH PLANNING TIME COMPARISON TABLE FOR DIFFERENT SIZE OF MAP

\begin{tabular}{|c|c|c|c|c|c|c|c|c|c|}
\hline \multirow{2}{*}{$\begin{array}{c}\text { Image } \\
\text { size }\end{array}$} & \multirow{2}{*}{$\begin{array}{c}\text { Thinning } \\
\text { time }\end{array}$} & \multirow{2}{*}{ start } & \multirow{2}{*}{ target } & \multicolumn{2}{|c|}{ thinning image } & \multicolumn{2}{|c|}{ original image } & \multirow{2}{*}{$\begin{array}{c}\text { Time } \\
\text { reduced }\end{array}$} & \multirow{2}{*}{$\begin{array}{c}\text { Increase of } \\
\text { path }\end{array}$} \\
\hline & & & & Path size & time & Path size & time & & \\
\hline \multirow{7}{*}{$200 * 200$} & \multirow{7}{*}{0.013453} & \multirow{7}{*}{$(0,32)$} & $(32,140)$ & 139 & 0.358289 & 139 & 0.364221 & $1.63 \%$ & $0 \%$ \\
\hline & & & $(68,199)$ & 232 & 0.377337 & 230 & 0.447982 & $15.6 \%$ & $0.86 \%$ \\
\hline & & & $(128,0)$ & 160 & 0.431301 & 159 & 0.530645 & $18.7 \%$ & $1.26 \%$ \\
\hline & & & $(98,70)$ & 135 & 0.344718 & 134 & 0.379968 & $9.3 \%$ & $0.75 \%$ \\
\hline & & & $(159,180)$ & 300 & 0.495573 & 298 & 0.677165 & $26.8 \%$ & $0.67 \%$ \\
\hline & & & $(180,90)$ & 235 & 0.755732 & 233 & 1.32665 & $43.1 \%$ & $0.86 \%$ \\
\hline & & & $(199,160)$ & 321 & 0.55126 & 320 & 0.560022 & $1.6 \%$ & $0.31 \%$ \\
\hline \multirow{7}{*}{$800 * 800$} & \multirow{7}{*}{0.287893} & \multirow{7}{*}{$(0,130)$} & $(126,545)$ & 543 & 0.960035 & 536 & 1.35808 & $29.3 \%$ & $1.31 \%$ \\
\hline & & & $(273,799)$ & 939 & 1.75971 & 918 & 2.64039 & $33.4 \%$ & $2.29 \%$ \\
\hline & & & $(513,0)$ & 642 & 1.9027 & 635 & 32.6216 & $94.2 \%$ & $0.76 \%$ \\
\hline & & & $(393,281)$ & 544 & 0.898026 & 530 & 1.1792 & $23.8 \%$ & $2.64 \%$ \\
\hline & & & $(635,725)$ & 1,225 & 2.95465 & 1,177 & 6.8273 & $56.7 \%$ & $4.07 \%$ \\
\hline & & & $(720,370)$ & 959 & 7.75812 & 936 & 67.5522 & $88.5 \%$ & $2.46 \%$ \\
\hline & & & $(799,638)$ & 1,303 & 2.86236 & 1,262 & 4.24947 & $32.6 \%$ & $3.32 \%$ \\
\hline
\end{tabular}

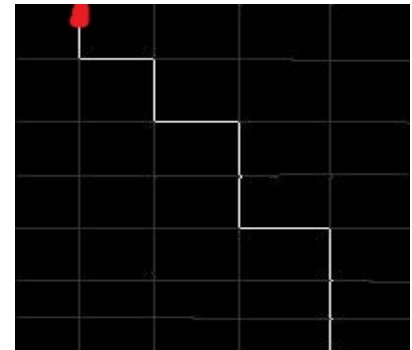

Figure 6. Path in the thinned map

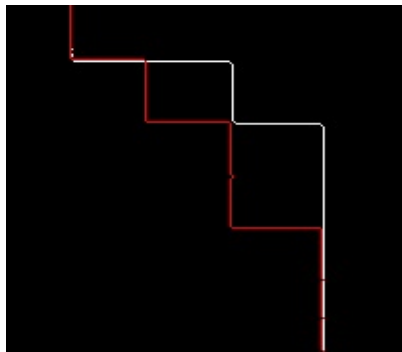

Figure 7. Thinned image

From the Table I : For planning path, it takes less time on grid map with thinning preprocess than in the original grid map. The larger the map is, the more obviously the time is reduced. The time even is reduced by more than $90 \%$ on the grid map of $800 * 800$. If the start and target points are not in the skeleton of the thinned map, the path will be longer. But it increases by no more than $5 \%$.

\section{B. Simulation -2 :The Different Complexity of Grid Map}

Figure 8 is a grid map of $400 * 400$ which adds the thinned map and Figure 11 shows the result of the thinned path and the original path with the key point reserved. These maps have the same size with the one in Figure 4 where path is planned. The path results are shown in the Figure 9, Figure 10 and the data results are shown in the Table II :

As shown in Table II , time is reduced by $90 \%$ when plan path on the map thinned in the Figure 10 than the original one in the Figure 9. However it is reduced by $70 \%$ when plan path on the map thinned in the Figure 6 than the original one in the Figure 5.

It proves that path planning algorithm with thinning preprocess can greatly reduce the searching time on the complicated environment. But this algorithm increases the length of the path to some extent. This algorithm increases the length of the path to some extent. But it increases by no more than $20 \%$.

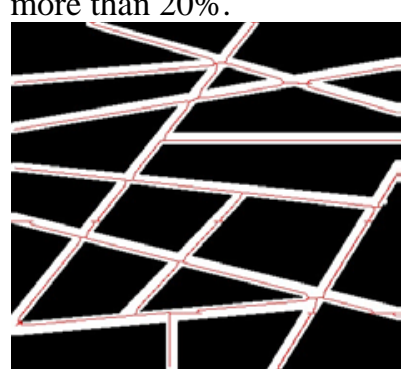

Figure 8. Map

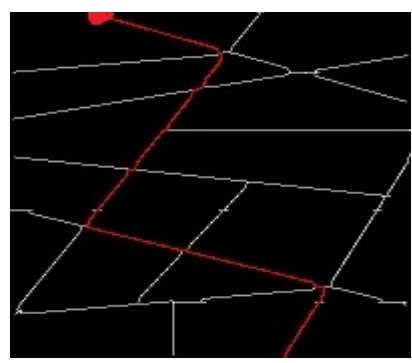

Figure 10. Path in the map

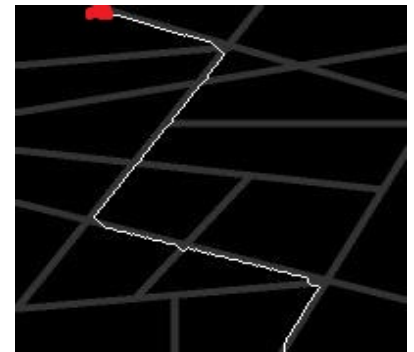

Figure9. Path in the map

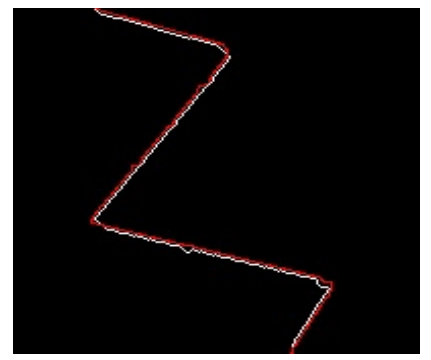

Figure 11. Path 
TABLE II. PATH PLANNING TIME COMPARISON TABLE FOR DIFFERENT COMPLEXITY OF MAP

\begin{tabular}{|c|c|c|c|c|c|c|c|c|c|}
\hline \multirow{2}{*}{ Image } & \multirow{2}{*}{$\begin{array}{c}\text { Thinning } \\
\text { time }\end{array}$} & \multirow{2}{*}{ start } & \multirow{2}{*}{ end } & \multicolumn{2}{|c|}{ thinning image } & \multicolumn{2}{|c|}{ original image } & \multirow{2}{*}{ time reduced } & \multirow{2}{*}{ Increase of path } \\
\hline & & & & path size & time & path size & time & & \\
\hline \multirow{5}{*}{$\begin{array}{l}\text { Figure } 4 \\
400 * 400\end{array}$} & \multirow{5}{*}{0.0569265} & \multirow{5}{*}{$(0,65)$} & $(63,279)$ & 278 & 0.485705 & 275 & 0.594361 & $18.30 \%$ & $1.09 \%$ \\
\hline & & & $(256,0)$ & 320 & 0.70814 & 318 & 2.46266 & $71.20 \%$ & $0.63 \%$ \\
\hline & & & $(196,141)$ & 271 & 0.5377 & 265 & 0.590478 & $8.90 \%$ & $2.26 \%$ \\
\hline & & & $(359,180)$ & 473 & 1.66013 & 464 & 6.4065 & $74.10 \%$ & $1.94 \%$ \\
\hline & & & $(399,319)$ & 649 & 0.99142 & 631 & 1.42847 & $30.60 \%$ & $2.85 \%$ \\
\hline \multirow{4}{*}{$\begin{array}{l}\text { Figure } 8 \\
400 * 400\end{array}$} & \multirow{4}{*}{0.104964} & \multirow{4}{*}{$(0,80)$} & $(71,294)$ & 219 & 0.396798 & 215 & 0.653822 & $39.30 \%$ & $1.86 \%$ \\
\hline & & & $(137,399)$ & 468 & 1.80445 & 406 & 38.8995 & $95.40 \%$ & $15.27 \%$ \\
\hline & & & $(334,162)$ & 536 & 3.10676 & 498 & 81.693 & $96.20 \%$ & $7.63 \%$ \\
\hline & & & $(399,272)$ & 669 & 6.49825 & 640 & 271.218 & $97.60 \%$ & $4.53 \%$ \\
\hline
\end{tabular}

From these two groups of experiments above, some conclusions are shown as below: (1) Path planning algorithm with thinning can improve the search efficiency of path planning. (2) On large or complicated map of the environments, path planning algorithm can reduce the time of searching more obviously.

\section{CONCLUSION}

In order to reduce the time of path planning, the path planning algorithm with thinning preprocess is proposed in this paper. Zhang's fast parallel thinning algorithm combined with $A^{*}$ algorithm verifies the feasibility of the algorithm in this paper. A modified Zhang's fast parallel thinning algorithm is proposed to reserve the key points which can be marked manually.

Two groups in this paper prove the validity of the path planning algorithm with thinning preprocess. And it can come to conclusion that the algorithm proposed in this paper has advantages include: (1) Reduce the search time of planning path. (2) Plan path for special start and target points with short time. (3) The effect is more obvious on large or complex map of the environments. The disadvantages include: (1) Thinned map with points reserved has more burrs. (2) The results of the path planning are suboptimal. The path that was planned out is longer if the start or target points are not in the skeleton of the thinned map.

Path planning algorithm with thinning preprocess can also be combined with other algorithms that can use grid map, such as genetic algorithm, ant colony algorithm and so on.

\section{REFERENCES}

[1] Qi Zhang. Path Planning And Location For Mobile Robot [D]. Harbin Institute of Technology,2014.

[2] C. Zeng, Q. Zhang, X.Wei, "GA-based global path planning for mobile robot employing A* algorithm,” Journal of Computers, vol. 7, no. 2, pp. 470-474, Feb. 2012.

[3] Q. Yao-Hong, P. Quan and Y. Jian-Guo, "Flight path planning of uav based on heuristically search and genetic algorithms", Thirty-First Annual Conference of the IEEE Industrial Electronics Society, 2005.

[4] ALI S, BRIAND L C, HEMMATI H. Modeling robustness behavior using aspect-oriented modeling to support robustness testing of industrial systems [J]. Software \& Systems Modeling,2012, 11(4):100-115.

[5] Na Wang. Mobile Robot Topological Map Building Based on Thinning Algorithm [D]. Shandong University,2009.

[6] Chang-Hyuk Choi, Jae-Bok Song, Woojin Chung and Munsang Kim,"Topological map building based on thinning and its application to localization," Intelligent Robots and Systems, 2002. IEEE/RSJ International Conference on, 2002, pp. 552-557 vol.1.

[7] Liang Yu, Guangwei Wang. Fast Thinning Algorithm For Binary Map [J]. Science And Technology Vision,2012,14:111-112+100

[8] Ying Bai. Study of the hardwritten Chinese character's thinning algorithm [D]. Xidian University,2014.

[9] Lei Zhao, Qiong Chen, Zhong Chen. A new improved OPTA thinning algorithm [J]. Computer application,2008,10:2639-2642.

[10] Xuanzhong Wu. Extending of Zhang's fast parallel thinning algorithm [J]. Journal of Fujian University of Technology,2006,01:89-92. 